\title{
Routing Protocols for Energy Efficiency in WSNs: A review
}

\author{
Dheyab Salman Ibrahim, Abd Ali Hussein, Fadhil Kadhem Zaidan \\ Department of Computer, Science College, University of Diyala, Diyala, Iraq
}

\begin{abstract}
Wireless sensor networks (WSNs) is a significant part of networking area. They are cheap, efficient, and small, they are become more and more important for several applications. However, with the widespread use of applications that rely on wireless sensor networks, they continue to suffer from limitations such as data routing and security. WSNs can be used for several applications. Nodes of Sensor work on batteries. It is impossible to charge or replace the battery in some applications. For that purpose, optimal use of node's energy is a key issue in the network. Routing is a significant challenge to extend the lifespan of network. Several protocols are implemented in WSNs. In this study, a survey on clustering techniques, present a characteristic and requirements routing design of WSNs. Result of this study shows how to solve some of these problems. WSN routing protocols have to ensure shortest paths, minimum energy consumption, less delay and maximum lifespan of network.
\end{abstract}

Keywords - WSN; Energy Efficiency; Clustering; Cluster Members; Cluster Head.

\section{Introduction}

WSN consists of a set of big number of sensor nodes organized in ad hoc form to interact with the physical world. Each node involves some elements: "sensors,

DOI: 10.18421/SAR41-05

https://doi.org/10.18421/SAR41-05

Corresponding author: Dheyab Salman Ibrahim, Department of Computer, Science College, University of Diyala, Diyala, Iraq.

Email: dheyab.ibrahim@student.uobabylon.edu.iq

Received: 18 February 2021.

Revised: 15 March 2021.

Accepted: 19 March 2021.

Published: 22 March 2021.

(c) BY-NC-ND (C) 2021 Dheyab Salman Ibrahim, Abd Ali Hussein \& Fadhil Kadhem Zaidan; published by UIKTEN. This work is licensed under the Creative Commons Attribution-NonCommercial-NoDerivs 3.0 License.

The article is published with Open Access at www.sarjournal.com controllers, power supply and transceivers" [1]. Sensor nodes may be distributed regularly or randomly (installed manually). The area of sensing is the area where sensors are deployed.

Sensors collect the data from the interest region, handle it, and transmit these data to base station (BS) (also called sink) or to other nodes wirelessly [2].

WSNs have constraints such as limited amount of energy, small communication scope, low bandwidth, and limited processing. Environment is the crucial issue in defining: size, distribution scheme, and topology of the network [3].

Energy consumption is considered as a key factor in WSN design. When sensor nodes are deploying in complex environment, it is very hard ("or not possible") to substitute or recharge batteries. The cost of transmission unit is higher than sensing and processing in WSNs. So, energy saving through the best strategy for data transmission is needed to redirect data from nodes to $\mathrm{BS}$ to extend the network lifetime [1]. Clustering is important technique for energy efficiency. In this method, nodes are structured into clusters. The nodes in cluster are called as "cluster members" and selected among them Cluster Head (CH) [1].

This paper is structured as follows. Section 1 is introduction of the WSNs. Section 2 shows WSNS components. Section 3 presents Routing Protocols in WSNs. Section 4 presents Clustering Algorithms. Section 5 is about General Architecture of Clustering. Section 6 presents Discussion. Section 7 is about Motivation. Section 8 describes Challenges. Section 9 is the Conclusion.

\section{Wireless Sensor Network Components}

The basic element in wireless sensor node consists of four major units: sensing unit, computation unit, communication unit, and power unit. In addition, there are three optional units, which can be combined with the sensor node such as: localization system, mobilizer, and power generator. Figure 1 shows the parts of a typical wireless sensor node [4]. 
1) Sensing Unit: It is responsible for sensing the physical phenomena and produces the analog signals to the ADC so as to convert it to digital data, and sends it to the computation unit.

2) Computation Unit: This unit is used to manage and manipulate the instructions that are related to sensing, communication, and self-organization. It is composed of a processor chip, an active shortterm memory for storing the sensed data, an internal flash memory for storing program instructions, and an internal timer.

3) Communication Unit: It is responsible of all data transmission and reception of the sensor node that are performed by the transceiver circuitry.

4) Power Unit: This unit represents the most significant part in wireless sensor node. It supplies the other units by the needed power.

Also another component within WSNs, such as a localization system, is a power generator and a mobilizer.

\section{a. Localization System}

It is important that the node of wireless sensor worked with a location finding system since it is necessary for many WSN applications. It is required by routing algorithms and sensing coverage algorithms, which need information about the location of the nodes of wireless sensor. The location finding system is composed of a Global Positioning System (GPS) or a discovery algorithm that executes localization systems to provide information about the location of wireless sensor node using distributed computation.

\section{b. Mobilizer}

The mobility function is sometimes needed in many applications to move the wireless sensor node from one location to another so as to perform a certain task in WSN, so it will be necessary that the wireless sensor node is equipped with the mobilizer system for such applications. A high energy consumption is needed to support the mobility in wireless sensor node, and it should be supported efficiently. The movement of wireless sensor node is controlled by the mobility function with cooperation with the sensing unit and the computation unit.

\section{c. Power Generator}

Several WSN applications need to operate for a longer time, so it is essential to equip the wireless sensor node with additional power source in order to prolong the network lifetime.

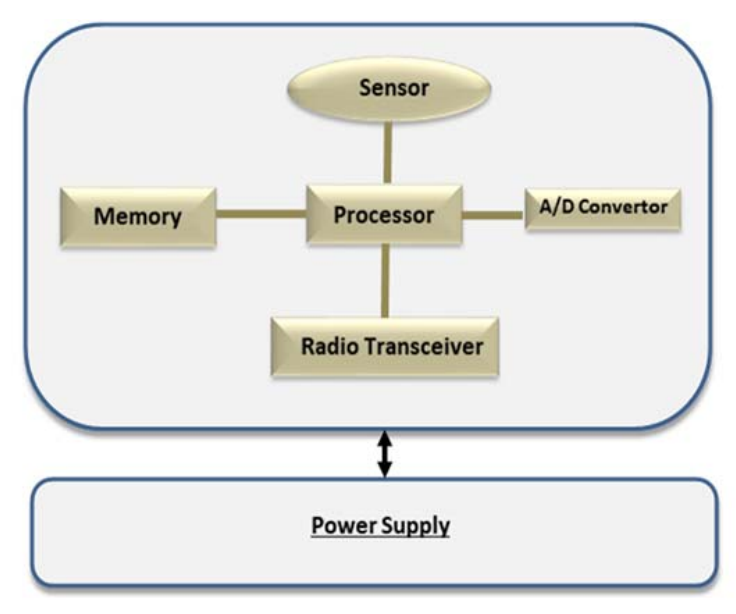

Figure 1. Components of WSNs

\section{Related Works}

Routing is a method for route between source and destination nodes. Key objective of routing protocols is to determine the shortest path that bring efficient energy consumption, less delay, the best service in order to expand the network lifetime via saving the sensors node alive more times as possible. [5] Discussed the use of secure routing protocol to enhance security of network. Most routing protocols are work with some failures within network and may have insecurity. In this study we suggest secure routing technique with using multi data flow topologies (MDT) scheme to protect from threats, and we suggest optimization methods to decrease the energy cost, too. [6] discussed an effective routing solving is distinguished via enabling the increase lifetime of the network, which improves efficiency and guarantee the best quality of service. [7] Present the routing method with energy efficient, mobile sinks and split network to several clusters. They analyze the influence of mobile sink number on lifetime of the network and show the results of the simulation display that network has top execution in around 3 of mobile sink. [8] Gives taxonomy for routing based clustering protocols and examines the hybrid hierarchical routing protocol that is distinguished with intelligent routing protocols. Proposed usage swarm intelligence in cluster creation and their hierarchical routing to improve the scalability and robust of hierarchical routing protocols is explained in [9]. In this study routing protocol is described named "Delay and energy efficient proactive" for WSNs (DEEP) which is a hierarchical kind for shortest path and a clustering method to do aggregation. The protocol targets to provide trade-off between delay and energy consumption. [10] Discussed all routing protocols applied in WSNs. In [11] Genetic Algorithm (GA) is Proposed to optimize and determine the routes among the nodes to/from the sink, decrease the energy costs. In [12], Network structure and node 
mobility protocols are classified and the design requirements for routing protocols are analyzed. [13] A discussion that routing protocols play an important role in ensuring efficient communication between the source and destination nodes. Selection of the best routing protocol provides good efficiency, operation, and reliability of the network. So, WSN routing protocols can be categorized and based on network structure into three major sets [18]:

Flat routing protocols, hierarchal routing protocols and location-based protocols.

\section{Clustering Algorithms}

Clustering method in WSNs is the solutions to improve energy efficiency and extended network lifetime. It decreases amount of messages communicated to the sink. Clustering is the choice which presents a group of nodes in the network to create good topology. This method gives an effective network organization. [15] studied the choice of the Cluster Leader to improve the life span of the WSN. When selecting a Cluster Leader (CL), one can see the energy of the rest nodes and select the maximum energy nodes in each cluster and prohibition nodes, i.e. those with poor energy. [16] introduced a cluster based adaptive duty cycle hybrid MAC protocol ADMAC for WSNs. In this protocol, cluster heads $(\mathrm{CH})$ are spread in $\mathrm{L}$ phases to decrease idle listening time. This will decrease energy consumption from idle listening and collision. [17] Present efficient cluster head selection scheme switches the "cluster head" position between the nodes that have higher energy level compared with other. The algorithm counts initial energy, residual energy and an optimum value of cluster heads in order to select the following set of cluster heads of the network that fits for IoT applications for example, smart cities, environmental monitoring. In [18], "Sensor Nodes" link with each other by routing protocols are Presented. Routing Protocols can be categorized in several kinds in WSNs. Here, one should focus on the Hierarchical (cluster-based) routing protocols and study their performance by simulating tools. [19] Presented the task that show "how to select cluster heads" and "route data" via energy-efficient paths to destination. [20] Proposed "Hybrid" Multi-hop based clustering routing protocol that is suitable for exact environment and the needs, and it balances the network's lifetime. HMPBC is used to reduce number of forwarding via picking cluster-heads in the procedure of data communication and to decrease energy consumption. [21] Proposed clustering scheme is based on distributed method, multi-hop transmission and displays the proposed system with improved performance of energy consumption.

\section{Characteristics of WSNs and Design Objectives}

Sensor networks have several characteristics and requirements in term of network performance and capabilities [22].

WSNs have several characteristics compared to the classic wireless communication networks:

- Deployment Techniques;

- Battery-powered;

- Severe energy, computation, and storage constraints;

- Self-configurable;

- "Many-to-One" traffic;

- Topology change.

Sensor networks have different application requirements:

- Small size of node;

- Low cost;

- Low power consumption;

- Scalability;

- Reliability;

- Fault tolerance;

- Security.

\section{Discussion}

The key aim of this paper is to provide the latest idea of the structure of WSNs, challenges of WSNs, Routing Algorithms and Clustering Methods. This study analyzes WSNs by surveying previous studies on this topic. We provide a survey of these articles to support future researchers who explore this area. Regarding a number of researchers concerned in IoTbased WSNs technologies, we perceive that there are lacks such as an impaired organization which leads to inappropriate research activities. A number of researches have been conducted on wireless networking of networks from a primary perspective, while other researchers have focused on developing WSNs capabilities. This study gives a clear research framework and management capacity, so this provides researchers with important research ideas related to the most important problems and solutions in this field. This study categorizes WSNs based on their limitations that become the interest of researchers who also want to motivate customers and users' attention in this area. This study can help the researchers discover gaps and weaknesses in the literature.

\section{Motivations}

The components of communication for WSNsbased technologies have become a promising study field. This part surveys a group of related studies that 
show the advantages of routing, clustering, energy efficiency of WSNs, which is integrating with the Internet and with cloud computing, which is needed in IoTs. So the main motivation for this study is to give more attention for the following requirements [14]: energy efficiency, quality of service, scalability and reliability.

\section{Challenges and Related Recommendations}

In this section, we discuss the "limitations" of WSNs and make some suggestions to improve the efficiency of the sensor networks. There are some "challenges" in the implementation of WSNs. While WSNs have many advantages, they do not provide the best solution for connecting WSNs to the Internet or IoTs components. Academics and researchers face a lot of problems or limitations when employing the WSNs technologies for different applications in order to monitor environment with the IoTs.

Some main challenges for Energy efficiency are to reduce energy consumption due it is more necessary to span battery life. They are defined in the following:

1. WSNs continue to need high power from energy constrained batteries for data processing and sending.

2. The nodes in WSNs have limited memory and computational capabilities. So, another challenge for WSNs depends on applications.

3. Routing protocols and design objectives. [5-14].

4. Clustering algorithms and cluster head election techniques. [15-22].

Due to these challenges, and to reduce energy consumption, we propose some recommendations for enhancing the performance of the WSNs:

$\checkmark$ Using specific methods to reduce the consumption of resource for the nodes of WSNs, such as, to develop algorithms or techniques to get optimization.

$\checkmark$ Using the effective computing and communication methods for the dissimilar applications of the WSNs that needs different "QoS", for instance, time restriction.

$\checkmark$ Improve Sensor deployment.

$\checkmark$ Improve Data Aggregation Techniques.

$\checkmark$ Improve Clustering mechanisms.

$\checkmark$ Improve Routing Protocols.

\section{Conclusion}

Due to the growth of technology WSNs becoms more important, and the sensors are nowadays employed in many environments. The limitations with WSNs become very complex to be capable to utilize. The outcomes for these limitations become essential goals for researchers. This study is done on the exiting aricles in order to survey imporant fiels in WSNs area displaying the main problems and the solutios as a suggestion to deal with these problems. We slso presented the key challenges that face WSNs technologies.

The main aim of this study is to provide researchers with more clarity and understanding and to draw the attention of researchers and specialists in this field to many aspects and fields and to a range of weaknesses and strengths.

\section{References}

[1]. Arjunan, S., \& Pothula, S. (2019). A survey on unequal clustering protocols in wireless sensor networks. Journal of King Saud University-Computer and Information Sciences, 31(3), 304-317.

[2]. Pathak, A., \& Tiwari, M. K. (2018, October). Clustering in wireless sensor networks based on soft computing: A literature survey. In 2018 International Conference on Automation and Computational Engineering (ICACE) (pp. 29-33). IEEE.

[3]. Bhende, M., Wagh, S. J., \& Utpat, A. (2014, April). A quick survey on wireless sensor networks. In 2014 fourth international conference on communication systems and network technologies (pp. 160-167). IEEE.

[4]. Kocakulak, M., \& Butun, I. (2017, January). An overview of Wireless Sensor Networks towards internet of things. In 2017 IEEE 7th annual computing and communication workshop and conference (CCWC) (pp. 1-6). IEEE. DOI: 10.1109/CCWC.2017.7868374

[5]. Patil, B., \& Kadam, R. (2018, January). A novel approach to secure routing protocols in WSN. In 2018 2nd International Conference on Inventive Systems and Control (ICISC) (pp. 1094-1097). IEEE.

[6]. El Hajji, F., Leghris, C., \& Douzi, K. (2018). Adaptive routing protocol for lifetime maximization in multi-constraint wireless sensor networks. Journal of Communications and Information Networks, 3(1), 67-83.

[7]. Zhong, P., \& Ruan, F. (2018, March). An energy efficient multiple mobile sinks based routing algorithm for wireless sensor networks. In IOP Conference Series: Materials Science and Engineering (Vol. 323, No. 1, p. 012029). IOP Publishing. 
[8]. Mehta, D., \& Saxena, S. (2018, August). A comparative analysis of energy efficient hierarchical routing protocols for wireless sensor networks. In 2018 4th International Conference on Computing Sciences (ICCS) (pp. 53-58). IEEE.

[9]. Argoubi, S., Maalaoui, K., Saidane, L. A., \& Boussada, R. (2018, June). Deep: Delay and energy efficient proactive routing protocol for event-driven wsns. In 2018 14th International Wireless Communications \& Mobile Computing Conference (IWCMC) (pp. 696-701). IEEE.

[10]. Singh, R., Kathuria, K., \& Sagar, A. K. (2018, December). Secure routing protocols for wireless sensor networks. In 2018 4th International Conference on Computing Communication and Automation (ICCCA) (pp. 1-5). IEEE.

[11]. Abro, A., Zhongliang, D., Memon, K. A., \& ul Ain, N. (2019, July). Novel Genetic Algorithm with Efficient Routing Paradigm for Multi-hop WSNs. In 2019 IEEE 9th International Conference on Electronics Information and Emergency Communication (ICEIEC) (pp. 28-31). IEEE.

[12]. Wang, R., He, G., Wu, X., Wang, F., \& Hu, Y. (2017, December). Research on routing protocols in wireless sensor networks with mobile sink. In 2017 IEEE 2nd Information Technology, Networking, Electronic and Automation Control Conference (ITNEC) (pp. 44-48). IEEE.

[13]. Shabbir, N., \& Hassan, S. R. (2017). Routing protocols for wireless sensor networks (WSNs). Wireless Sensor Networks-Insights and Innovations.

[14]. Koskela, P., Valta, M., \& Frantti, T. (2010). Energy efficient MAC for wireless sensor networks. Sensors \& Transducers, 121(10), 133.
[15]. Chitralingappa, P., \& Reddy, V. R. (2016). Cluster Leader Selection Using M-Leach-Based Routing Protocol For Efficient Energy In Wireless Sensor Network. EPRA International Journal of Multidisciplinary Research (IJMR), 2013, 6.

[16]. Priya, B., \& Manohar, S. S. (2020). Adaptive Power Control and Duty Cycle based Medium Access Control Protocol for Cluster based Wireless Sensor Network. Science And Technology, 23(1), 38-54.

[17]. Behera, T. M., Mohapatra, S. K., Samal, U. C., Khan, M. S., Daneshmand, M., \& Gandomi, A. H. (2019). Residual energy-based cluster-head selection in WSNs for IoT application. IEEE Internet of Things Journal, 6(3), 5132-5139.

[18]. Aya Ayad Hussein, Rajaaalden Abd Khaled, "Performance Analysis of Chain-Cluster based Routing Protocols in WSN ",IOSR Journal of Electronics and Communication Engineering (IOSRJECE), Volume 15, 2020.

[19]. Ghaddar, A., Ghosn, M. H., \& Mitton, N. (2020, May). R-MUCH: A Clustering Routing Algorithm Using Fuzzy Logic for WSNs. In IOP Conference Series: Materials Science and Engineering (Vol. 853, No. 1, p. 012049). IOP Publishing.

[20]. Wang, C., Zhang, Y., Wang, X., \& Zhang, Z. (2018). Hybrid multihop partition-based clustering routing protocol for WSNs. IEEE Sensors Letters, 2(1), 1-4.

[21]. Yousif, Y. K., Badlishah, R., Yaakob, N., \& Amir, A. (2018, June). An energy efficient and load balancing clustering scheme for wireless sensor network (WSN) based on distributed approach. In Journal of Physics: Conference Series (Vol. 1019, No. 1, p. 012007). IOP Publishing.

[22]. Singh, S. K., Singh, M. P., \& Singh, D. K. (2010). Routing protocols in wireless sensor networks-A survey. International Journal of Computer Science \& Engineering Survey (IJCSES), 1(2), 63-83. 